\title{
Research on Technology of Azimuthal Electromagnetic Wave Resistivity logging While Drilling and Its Application in Geosteering
}

\author{
Hexin $\mathrm{Li}^{1, \mathrm{a}}$, Yongshou DAl ${ }^{1}$, Weining $\mathrm{Ni}^{2}$, Weifeng Sun ${ }^{1}$, Ligang $\mathrm{Li}^{1}$, \\ Xiaobin Zhang ${ }^{1}$ \\ ${ }^{1}$ School of Information and Control Engineering, China University of Petroleum, Qingdao 266580, \\ China; \\ ${ }^{2}$ SINOPEC Research Institute of Petroleum Engineering, Beijing100101, China.
}

a329575054@qq.com

Keywords: electromagnetic wave resistivity, geosteering, orientation imaging, MWD.

\begin{abstract}
With the number of old oil fields exploited by horizontal well increased, the complexity and difficulty to extract oil from reservoir geology promote the further development of logging while drilling (LWD) technology. Thus, the LWD technology develops from the initial no-directional shallow measurements to current deep directional measurements. Based on electromagnetic wave propagation theory, electromagnetic wave resistivity logging while drilling technology is adopted to capture the resistivity from formation in real time. According to the polarization angles phenomena of resistivity curve, formation boundary can be detected. On the basis of conventional electromagnetic wave resistivity LWD, Schlumberger, Halliburton, Baker Hughes and GWDC have already carried out the research on azimuthal electromagnetic wave resistivity LWD measurement, and developed different kinds of azimuthal resistivity tools respectively, which can reflect the formation properties through $360^{\circ}$ real-time orientation imaging technology directly. In this paper, the operating principles and characteristics of four different kinds of azimuthal resistivity tools are introduced in detail, which helps to point out the research direction on technology of azimuthal electromagnetic wave resistivity logging while drilling and application in geosteering in future.
\end{abstract}

\section{Introduction}

The logging while drilling (LWD) technology, which can provide geosteering service, is being more widely to satisfy the requirement of petroleum exploration. And the azimuthal electromagnetic wave resistivity logging while drilling tool is a kind of important LWD tools, the transmitting and receiving coils of which mount on the drilling collar. It can infer spatial and temporal distribution of the electromagnetic field characteristics to identify resistivity of different formations by measuring amplitude attenuation EATT and phase differences $\Delta \Phi$ of the receiving coils while drilling. Then formation information could be acquired to realize geosteering and formation evaluation.

The conventional electromagnetic wave resistivity logging tool is made of axial transmitting and receiving coils [1]. It can accomplish multi-depth measurement through multi-frequencies and multi-spacing of transmitters-receivers under complicated geological conditions. Due to lack of orientation, the tool is not sufficient to be applied in anisotropic formation evaluation before drilling. In order to achieve better geosteering, the new tool called azimuthal electromagnetic wave resistivity while drilling tool which is based on conventional logging tool with horizontal or tilted antennas is developed [2]. Not only does it evaluate the formation efficiently, but also it achieves geosteering through $360^{\circ}$ real-time orientation imaging logging effectively. This paper summarizes the azimuthal electromagnetic wave resistivity LWD technology in details, including basic principles of logging technology and the application of geosteering. The differences between four major domestic and foreign logging tools are analyzed,it provides theoretical foundation for further research on azimuthal electromagnetic wave resistivity while drilling technology. 


\section{Azimuthal Electromagnetic Logging Principle Analysis}

We focus on the basic structure of electromagnetic wave resistivity LWD tool which has a transmitting coil and two receiving coils [3]. As shown in Figure 1, the transmitting coil, the two receiving coils are denoted as $T, R_{1}$ and $R_{2}$ respectively. The distance from the midpoint of $R_{1}$ to transmitting coil $T$ is $L_{1}$, and from the midpoint of $R_{2}$ to the transmitting coil $T$ is $L_{2}$, which satisfies $\mathrm{L}_{1}>\mathrm{L}_{2}$, the electromotive forces of Receiving coil $\mathrm{R}_{1}$ and Receiving coil $\mathrm{R}_{2}$ are $\mathrm{V}_{1}$ and $\mathrm{V}_{2}$ respectively.

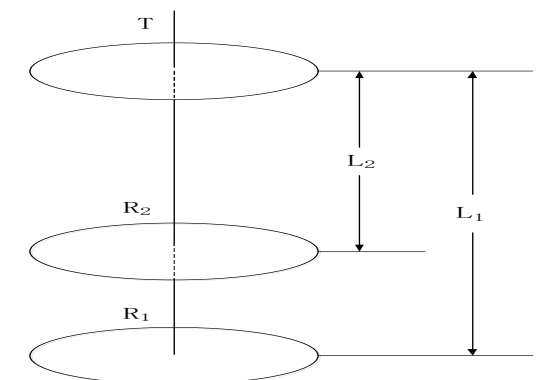

Fig. 1 The structure diagram of electromagnetic resistivity logging tool

Based on electromagnetic wave propagation theory, the transmitting coil $\mathrm{T}$ is supplied with stable alternating current, and then the current produces alternating electric field around wellbore (called primary electromagnetic field). Due to the alternating electric field, the toroidal vortex is induced in the formation which can build secondary alternative electromagnetic field, and then the receiving coils $R_{1}$ and $R_{2}$ receive different inductive electromotive forces $V_{1}$ and $V_{2}$. Because of the amount of amplitude attenuation EATT and phase difference $\Delta \Phi$ of $\mathrm{V}_{1}$ and $\mathrm{V}_{2}$ are related to electrical parameters (resistivity $\sigma$, permittivity $\varepsilon$ and permeability $\mu$ ), we can compute formation electrical parameters from EATT and $\Delta \Phi$.

Influenced by electrical parameters [5], therefore, the inductive electromotive forces of receiving coils can be expressed as

$$
\mathrm{V}_{\mathrm{i}}=\frac{\mathrm{C}_{\mathrm{i}} \mathrm{e}^{-\beta \mathrm{L}_{\mathrm{i}}+j \alpha \mathrm{L}_{\mathrm{i}}}}{\mathrm{L}_{\mathrm{i}}^{3}}\left[\alpha \mathrm{L}_{\mathrm{i}}+\mathrm{j}\left(1+\beta \mathrm{L}_{\mathrm{i}}\right)\right]
$$

Where $\mathrm{i}$ is the number of receiving coils, it takes one, two, three and so on, and $\mathrm{C}_{\mathrm{i}}$, and are other correlation parameters, they can be represented as

$$
\begin{aligned}
\mathrm{C}_{\mathrm{i}} & =\frac{\omega \mu \mathrm{S}_{\mathrm{Ri}} \mathrm{n}_{\mathrm{Ri}} \mathrm{M}}{2 \pi} \\
\alpha & =\omega \sqrt{\frac{1}{2} \mu\left[\sqrt{\varepsilon^{2}+\frac{\sigma^{2}}{\omega^{2}}}+\varepsilon\right]} \\
\beta & =\omega \sqrt{\frac{1}{2} \mu\left[\sqrt{\varepsilon^{2}+\frac{\sigma^{2}}{\omega^{2}}}-\varepsilon\right]}
\end{aligned}
$$

Where $\omega, \mu, \varepsilon, \sigma, M, S_{R i}$ and $n_{R i}$ are the current angular frequency, permeability, permittivity, moment of magnetic couple, area of receiver coil, and coil loop numbers, respectively.

The EATT are the amplitude attenuation of inductive electromotive forces, they can be expressed as

$$
\mathrm{EATT}=20 \lg \frac{\left|\mathrm{V}_{\mathrm{i}+1}\right|}{\left|\mathrm{V}_{\mathrm{i}}\right|}
$$

Where $\left|V_{i}\right|$ is the modulus of induction electromotive force of $V_{i}$.

The $\Delta \Phi$ are the phase differences of inductive electromotive forces, they can be expressed as

$\Delta \Phi=\Phi_{\mathrm{i}}-\Phi_{\mathrm{i}+1}$

Where $\Phi_{\mathrm{i}}$ is the phase angle of induction electromotive force of $\mathrm{V}_{\mathrm{i}}$. 
Combining (1), (2), (3), (4), (5)and(6),we can calculate the electrical parameters of resistivity $\sigma$, permittivity $\varepsilon$ and permeability $\mu$.

Generally, the electromagnetic well logging source frequencies always range from $0.19 \mathrm{~Hz}$ to $3.6 \mathrm{MHz}$, the dielectric constant can be considered as a constant because of low effect, and the permeability $\mu$ generally uses permeability of vacuum in oil and gas exploration. Therefore, the EATT and $\Delta \Phi$ are mainly affected by the formation resistivity $\sigma$, and Figure 2(a) shows the relationship between EATT and $\sigma$, while Figure 2(b) shows the relationship between $\Delta \Phi$ and $\sigma$.
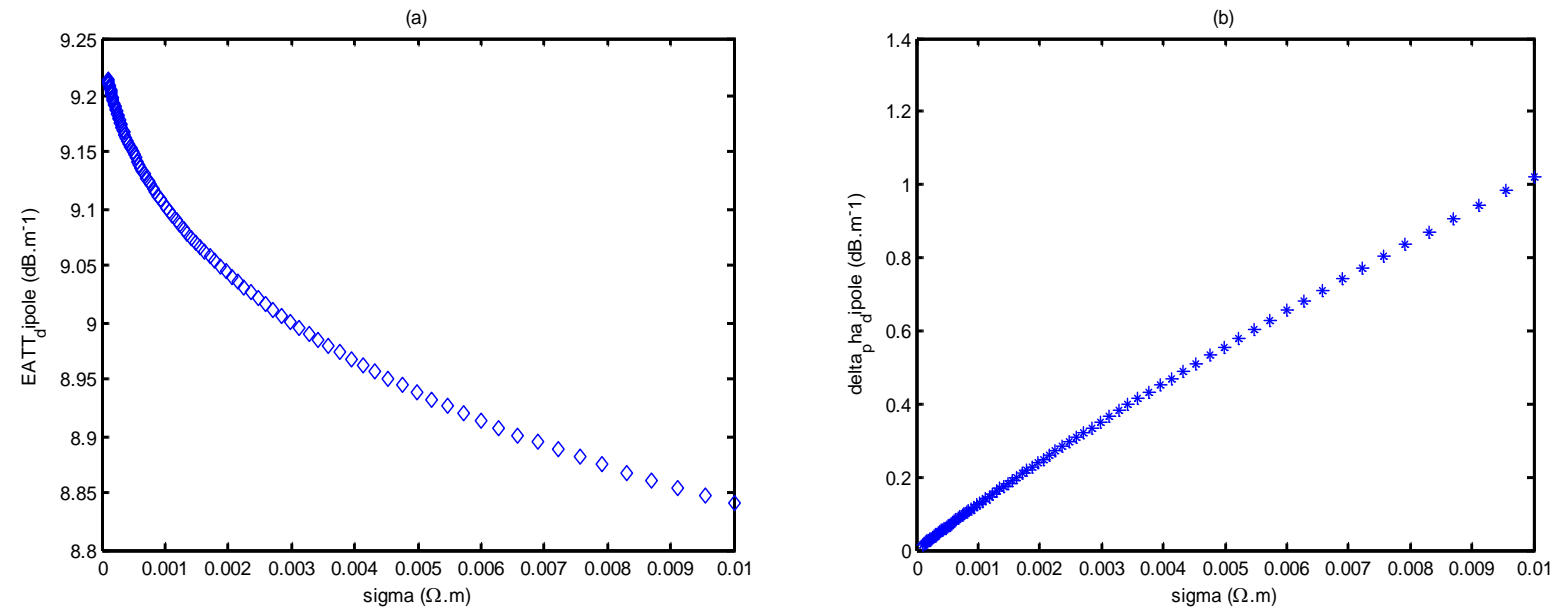

Fig. 2(a) The relationship between the EATT and $\sigma$; (b) The relationship between $\Delta \Phi$ and $\sigma$

We can calculate the true formation resistivity by $\Delta \Phi$ and EATT through a series of conversions, inversions, corrections, and then put it into use for geosteering and stratigraphic analysis. But the conventional electromagnetic wave resistivity logging tool is made of the axial transmitting and receiving coils, it is not only insensitive for orientation, formation anisotropy and other geological characteristics, but also not complete accurate measurement of high angle wells or horizontal wells.

So the relative angles among transmitting coils and receiving coils are changed in conventional tools, we talk about it as" the azimuthal electromagnetic wave resistivity logging tool”, which is similar to the conventional electromagnetic wave resistivity logging tool in measuring principle. When the tool goes through anisotropic formation, it can obtain inductive electromotive forces at the receiving coils. With the signal further decoupled and calculated, the directional resistivity measurements and azimuthal response value are obtained. So the tool both acquires the multi-probing depths and provides $360^{\circ}$ real-time imaging with the use of multi-frequencies, multi-angles of transmitters and receivers. And then using that information, we can forecast and judge the formation boundary, azimuthal stratigraphic characteristics and anisotropic formation as early as possible.

\section{The Development Of Azimuthal Logging Tools And Its Application}

\subsection{The Comparison of Azimuthal Logging Tools}

Currently, there are several representative azimuthal electromagnetic resistivity logging tools produced by Schlumberger's PeriScope 15(2005), Baker Hughes' AziTrak (2006), Halliburton's InSite ADR (2007) and GWDC's BWRX (2014). Different tools have different angles among the transmitting and receiving coils, the main differences among tools are shown in Table 1.

Table 1 Similarities And Differences Between Azimuthal Logging Tools

\begin{tabular}{cccccc}
\hline Tools & Coil Spacing / in & $\begin{array}{c}\text { Measurement } \\
\text { Frequencies / Hz }\end{array}$ & $\begin{array}{c}\text { Coils } \\
\text { Relationship }\end{array}$ & Imaging & $\begin{array}{c}\text { Conventional } \\
\text { Measurement }\end{array}$ \\
\hline PeriScope 15 & $22.0,34.0,44.0$ etc. & $\begin{array}{c}100 \mathrm{k}, 4000 \mathrm{k}, 2 \mathrm{M} \\
\text { Tilted Coils }\end{array}$ & $\begin{array}{c}\text { Yes } \\
\text { Orthogonal Coils }\end{array}$ & No & Yes \\
AziTrak & Single spacing & $400 \mathrm{k}, 2 \mathrm{M}$ & Yes \\
Insite ADR & $16.0,32.0,48.0$ etc. & $\begin{array}{c}125 \mathrm{k}, 500 \mathrm{k}, 2 \mathrm{M} \\
\text { Tilted Coils }\end{array}$ & Yes & Yes \\
BWRX & $34.3,44.0,45.8$ etc. & $500 \mathrm{k}, 2 \mathrm{M}$ & Tilted Coils & Yes & Yes \\
\hline
\end{tabular}


The Schlumberger electromagnetic resistivity logging tool of PeriScope15 is added two $45^{\circ}$ inclined coils and one $90^{\circ}$ tilted coil on the basis of ARC 5. The measurement signals of two $45^{\circ}$ inclined coils are used to detect the bed boundary, and the measurement signals of $90^{\circ}$ inclined coil are used to calculate the horizontal resistivity $R_{h}$, vertical resistivity $R_{v}$ and the inclination of reservoir in anisotropic formation [6].

Baker Hughes MWD integration tool AziTrak which is based on conventional drilling resistivity measurements has four transmitting coils, two receiving coils and two retrofitting horizontal receiving coils. The measurement signals of four transmitting coils and two receiving coils are used to measure resistivity, and the measurement signals of two retrofitting horizontal receivers which crossed with transmitting coils are used to detect the conductivity interface [7].

Insite ADR is Halliburton electromagnetic wave resistivity tool with six transmitting coils, two $45^{\circ}$ tilted receiving coils and a retrofitting transmitting coil. The six transmitting coils and two $45^{\circ}$ tilted receiving coils are symmetrical arranged, and the retrofitting transmitting coil has the same oblique angle with the receiving coils. The measurement signals of two $45^{\circ}$ tilted receiving coils can be used to measure azimuthal resistivity. Duo to the tilted coils, the tool has a deeper probing depth than before.

The azimuthal electromagnetic wave resistivity while drilling BWRX is developed by GWDC independently. It has four transmitting coils, two receiving coils and non-magnetic shell structure.

In summary, due to the different numbers of tilted coils, the azimuthal electromagnetic resistivity logging tools are different in aspects of investigation depths, azimuthal characteristics, vertical resolution and measurement ranges, and they can use the different resistivity curves and azimuthal logging images to geosteering.

\subsection{The Geosteering Used Resistivity Curve And Azimuthal Images}

The electromagnetic wave logging tool is based on the principle of mutual inductance [8]. When the tool is close to the formation boundary with a small angle, it produces electromagnetic waves which make the formation boundary generate capacitance effect, and then the boundary forms an additional electromagnetic field. The field is superimposed with the electric field which enhances the strength of the formation signal, and it leads to a sudden increase (far more than the true formation resistivity) and fast attenuation in resistivity curve. This phenomenon is known as the polarization angles of the resistivity curve. According to the existence of the polarization angles phenomenon, we can judge whether the tool is close to the boundary or not. No matter where the tool drills from the upper of surrounding rock to the target zone or from the target zone to the lower of surrounding rock, the resistivity curves have the same polarization angles ,and the width of polarization angles are also very narrow, so they cannot detect the specific azimuth of the tool.

In order to achieve better geosteerig, the azimuthal electromagnetic wave resistivity while drilling tool was designed with tilted transmitting or receiving coils on the basis of the conventional Electromagnetic Wave Resistivity logging tool. The tool obtains azimuthal detection capability, so it can achieve more accurate geosteering. It is different from conventional tool of high resistivity polarization angles when passing up and down the surrounding rock.

When the tool is in the middle of the target zone, the EATT and $\Delta \Phi$ measurement signals are zero. When it is in the high resistance target zone close to the upper part of the low resistance surrounding rock, the EATT and $\Delta \Phi$ measurement signals are positive. While approaching to the lower part of the low resistance of surrounding rock, the EATT and $\Delta \Phi$ measurement signals are negative, namely, the signals is the opposite in both cases. And when the distance between the tool and the formation interface is closer, the EATT signals are becoming larger and larger . If two sides of the formation boundary have greater difference in resistivity, the measurement signal will be stronger and the signal peak value will be bigger. Based on the above characteristics, the distance between the tool and formation boundary is obtained by the inversion algorithm. For the azimuthal logging tool, it can not only judge the direction through the signal curve of EATT and $\Delta \Phi$ measurement signals, but also reflect the formation property according to the real time $360^{\circ}$ imaging.

\subsection{The Application of Azimuthal Logging Tools}


The azimuthal electromagnetic wave measuring tools with tilted coils are able to indicate the existence of formation boundary as soon as possible. They can not only help to optimize the drilling trajectory, but also explain the geological features in real time, such as fracture, hole identification, the quantitative calculation of fracture and hole parameters through dynamic geological information, rapid interpretation information transmission and draw the well trajectory. In this paper, the applications of four kinds of logging tools are summarized.

Based on the adjacent wells condition, the PeriScope 15 is used to measure and forecast through data feedback and inversion calculation. The measured dates are processed through the ConocoPhillips device which is conducted by specialized geologists in special geological laboratory, and then the datas are transmitted back to PeriScope15. The PeriScope15 can be linked to EcoScope, GVR, TeleScope and other tools which provide the direction of boundary, the distance from target layer to formation boundary, annulus pressure, natural gamma ray, resistivity measurements and other information.

The AziTrak can simulate the intensity of received signal through inversion software RNS, with the comparison of the simulated values and measured values, we can get the distance of formation boundary. Then the measuring information of formation boundary is loaded into the geological software to scale and update the geological model timely.so we can use the tool to get azimuthal inclination, azimuthal gamma, resistivity, annulus pressure and vibration measurement.

The ADR comes together with the StrataSteer 3D geosteering service to provide a fully compensated, multiple-depth resistivity measurement, petrophysical evaluation and stratigraphic navigation solution in real time. The information is used by the Sperry's geosteering specialists to make immediate geosteering decisions for optimized wellbore placement and maximized reservoir exposure. And the well path will be delivered $100 \%$ in the target zone.

The BWRX firstly proposes a new method to provide the position and distance of formation boundary though the measurement signals of the cross linked coils. It can deliver azimuthal resistivity images in real time with the GW-LWD system software platform, and realize the accurate gesteering before drilling.

\section{Summary}

The azimuthal electromagnetic wave resistivity logging while drilling tool provides $360^{\circ}$ real-time imaging using multi-frequencies, multi-angles of transmitters and receivers, then we can use logging images to forecast the formation boundary before drilling and judge azimuthal stratigraphic characteristics in anisotropic formation as early as possible. But the tool has some limitations and needs for further improvement, such as (1) the resolutions of thin layer are low. (2) The geological features before drilling can't be measured. Therefore, how to improve the sensitivity of tool and detect the information before drilling will become research priorities in the future.

\section{References}

[1] Zhang K, Wei B J ,Chang L X, et al. Preliminary geo-steering method of directional electromagnetic wave resistivity logging-while-drilling tools. Progress in Geophysics.Vol.29 (2014) No.1, p.462-469.

[2] Wang G F, Luo X D, Hu S Q, Cui W M, Wu L H, Liu Q H, Wang X J, Sun C Y. Application of the azimuthal, deep-reading LWD to the to the optimization of geosteering in challenging North Sea well. Petroleum instruments. Vol.22 (2008) No.5, p.25-28.

[3] Yang Z, Yang J Z, Han L J. Numerical simulation and application of azimuthal propagation resistivity imaging while drilling. Journal of Jilin University: Earth Science Edition.Vol.43 (2013) No.6, p. 2035-2043.

[4] Gao J, Xin X Y, Chen W H, Chen P. Resistivity derivation in electromagnetic wave propagation resistivity logging while drilling. Well logging technology. Vol. 32(2008) No.6, p.503-507. 
[5] Yang J Z. Analysis on the affecting factors of prediction interface with azimuthal LWD electromagnetic tool. Well Logging Technology.Vol. 38(2014) No.1, p. 39-45.

[6] Song D W, Cao M Q, Zhang J Q, Wang X P. Application of PeriScope logging while drilling imaging technology for horizontal wells. Foreign oil field engineering.Vol.26 (2010) No.4, p.45-47.

[7] Wu Y M, Xiong S Q, Li C Y, Fang Y J. Application of AziTrak tool to geosteering of horizontal well development. Well logging technology. Vol.37(2013) No.5, p.547-551.

[8] Yang Z,Liu Q C, Yue B J, Liu H Y, Ma H B. On mechanism of polarization angle of electromagnetic logging while drilling and its influence factors simulation. Well logging technology. Vol.34(2010) No.3, p.210-214.

[9] Zhang W B, Wen Q B, Yang H, Xie L K. Techniques for horizontal well drilling in thin reservoirs of Luliang oilfield in Junggar Basin. Journal of oil and gas technology. Vol.33 (2011) No.4, p.112-114.

[10] Hao Y L, Du Z Q. Application of geosteering service based on OnTrak MWD/LWD platform in Jidong Oilfield. Well logging technology. Vol.33(2008) No.2, p.148-152.

[11] Xu F L, Lin N, Yang J Z, Gao X R. The un-drilled formation predicting technique with the azimuth electromagnetic wave resistivity while drilling. Mud logging engineering. Vol.22(2011) No.2, p.14-17. 